DOI: $10.1051 / \mathrm{rfm} / 2010003$

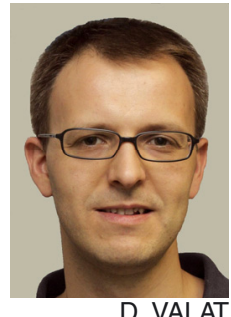

D. VALAT

\title{
Exactitude et stabilité du Temps atomique français TA(F)
}

\section{Accuracy and stability of the French atomic time scale TA $(F)$}

\author{
David VALAT, Michel ABGRALL et Pierre UHRICH
}

LNE-SYRTE (LNE, CNRS, UPMC), Observatoire de Paris, 61 Avenue de l’Observatoire, 75014 Paris, France, David.Valat@obspm.fr

\section{Résumé}

Le Temps atomique français TA(F) est une échelle de temps calculée à partir de données d'étalons commerciaux à césium, entre vingt et trente horloges selon la période considérée, provenant de neuf institutions ou entreprises situées sur le territoire national. Le TA(F) est la référence nationale scientifique de temps avec des qualités d'exactitude et de stabilité de fréquence. Dans le double but de rapprocher l'intervalle unitaire de l'échelle TA(F) de la seconde du SI et d'améliorer sa stabilité de l'échelle, il a été décidé en 2002 de piloter cette échelle de temps, dont la marche était laissée libre jusque là, sur les étalons primaires de fréquence du LNE-SYRTE. La fréquence du TA(F) a dans un premier temps été corrigée mensuellement pour ramener lentement l'échelle de temps vers le Temps atomique international TAI. Cette correction a permis de compenser la dérive de l'échelle sans perturber sa stabilité à trente jours. Une fois suffisamment proche de la seconde du SI, la fréquence du $\mathrm{TA}(\mathrm{F})$ a été dans un deuxième temps pilotée mensuellement sur la fréquence moyenne des étalons primaires du laboratoire ; ce qui a permis l'amélioration de la stabilité à trente jours, avec un gain d'un facteur 2 par rapport à la situation précédente. L'article décrit le pilotage en fréquence du TA(F), c'est-à-dire comment les corrections ont été appliquées, et montre les résultats obtenus, en terme de stabilité relative par rapport au TAI, et en terme d'exactitude en comparant la fréquence du $\mathrm{TA}(\mathrm{F})$ à la seconde du SI sur le géoïde en rotation telle qu'elle est publiée dans la Circulaire $\mathrm{T}$ du Bureau international des poids et mesures (BIPM). Depuis juillet 2006, l'écart relatif de fréquence sur $30 \mathrm{~d}$ entre le TA(F) et la seconde du SI est pratiquement resté dans les limites de $1,8 \times 10^{-15}$. Ce chiffre matérialise les limites de l'accord entre l'intervalle unitaire du TA(F) et la seconde du SI, un résultat majeur qui n'a été rendu possible que par la mise en oeuvre opérationnelle de quatre étalons primaires de fréquence au laboratoire.

MOTS CLÉS : ÉCHELLES DE TEMPS, TEMPS ATOMIQUE FRANÇAIS, SECONDE DU SI.

\section{Abstract}

The French atomic time scale TA $(F)$, which is computed with data from 20 to 30 industrial caesium standards, depending on the given pe- riod of time, located in nine institutions or companies located on French soil, is aiming at providing a common stable national frequency reference to the contributing institutions. To improve its stability, it was decided in 2002 to steer it to the LNE-SYRTE Primary Frequency Standards (PFS). The frequency of TA $(F)$ was first slowly corrected monthly to compensate the drift without disturbing the $30 \mathrm{~d}$ relative frequency stability of the time scale. Once close enough to the SI second, the time scale was steered monthly to the frequency data issued from the LNESYRTE PFS, providing that way a more stable reference, improved by a factor 2 with respect to the former system. We describe the steering applied to $T A(F)$ and show the results in terms of relative stability with respect to TAI, or by comparing TA $(F)$ to the SI second on the geoid as published monthly by the BIPM in its Circular T. We apply this steering since July 2006. With this method, the departure over 30 d intervals of $T A(F)$ from the SI second on the Geoid is globally maintained inside the limits $1.8 \times 10^{-15}$. Within these limits, the TA $(F)$ scale unit interval is in agreement with the SI second, a result which was made possible thanks to the four PFS currently in operation in the LNE-SYRTE.

KEY WORDS: TIME SCALES, FRENCH ATOMIC TIME SCALE, SI SECOND.

\section{Glossaire}

Aces : $\quad$ Atomic clock ensemble in space, projet de l'Agence spatiale européenne de mise en orbite d'horloges atomiques à bord de la Station spatiale internationale.

Arima : Autoregressive integrated moving average.

BIH : Bureau international de l'heure, situé à l'OP jusqu'en 1986, devenu ensuite la Section du Temps du BIPM.

BIPM : $\quad$ Bureau international des poids et mesures, dont la Section du Temps calcule EAL, TAI, UTC et la seconde du SI sur le géoïde en rotation.

CNES : Centre national d'études spatiales, Agence française de l'espace. 
EAL : Échelle atomique libre, première étape du calcul de l'UTC par le BIPM.

EAL $(F)$ : Échelle atomique libre française, première étape du calcul de l'actuel TA(F) basée sur une moyenne pondérée des écarts à une modélisation Arima des horloges atomiques ; jusqu'au $1^{\mathrm{er}}$ janvier 2002, TA(F) est identique à EAL(F).

Euramet : Organisation régionale européenne de métrologie.

LNM : $\quad$ Laboratoire national de métrologie.

OP : $\quad$ Observatoire de Paris.

Pharao : Projet d'horloge atomique à refroidissement d'atomes en orbite, projet spatial du CNES faisant partie du projet Aces.

SYRTE : Systèmes de références temps espace, laboratoire de l'OP où est situé le LNM français pour les unités de temps et de fréquence, le LNE-SYRTE.

TA(F) : Échelle de temps atomique français, issue du pilotage de EAL(F) sur les étalons primaires de fréquence du LNESYRTE.

TAI : $\quad$ Temps atomique international, issue du pilotage de EAL par les étalons primaires de fréquence des LNM dont les données sont transmises au BIPM.

UAI : $\quad$ Union astronomique internationale.

UT1 : Temps universel, basé sur les observations astronomiques.

UTC : $\quad$ Temps universel coordonné, issu du TAI par l'ajout ou le retrait de secondes entières : en 2009, TAI - UTC $=34 \mathrm{~s}$.

UTC(OP) : Réalisation physique temps réel du futur UTC, réalisée par le LNE-SYRTE à l'OP.

\section{Introduction}

Le Laboratoire national de métrologie (LNM) pour les unités de temps et de fréquence, le LNE-SYRTE, intégré au laboratoire Systèmes de référence temps espace (SYRTE) à l'Observatoire de Paris (OP), a entre autres missions celle de générer deux échelles de temps : la réalisation en temps réel pour la France du Temps universel coordonné UTC, dénommée UTC(OP), et la génération d'une référence nationale de temps fréquence, le Temps atomique français $\mathrm{TA}(\mathrm{F})$. Cet article porte uniquement sur le TA(F) et sur l'amélioration de ses performances métrologiques au cours de ces 4 dernières années.

Cette activité de maintien et d'amélioration du TA(F) s'inscrit dans un contexte international précis qui est décrit à la section 2. Après un bref retour sur l'historique du $\mathrm{TA}(\mathrm{F})$ à la section 3 , on décrit, dans la section 4 son état actuel. Les sections 5 et 6 montrent les méthodes d'étalonnage de la fréquence du TA(F) mises en œuvre respectivement sur la base de Temps atomique international (TAI) puis sur la base des données des étalons primaires de fréquence du laboratoire. Une nette amélioration de la stabilité de fréquence au-delà d'une période d'analyse de $30 \mathrm{~d}$ est mise en évidence à la section 6. Enfin, la section 7 propose une validation de l'incertitude du raccordement en fréquence du $\mathrm{TA}(\mathrm{F})$ aux étalons primaires.

\section{Contexte international}

L'UTC est une échelle de temps « papier », c'est-àdire sans réalisation physique, calculée mensuellement par la section du Temps du Bureau international des poids et mesures (BIPM), à partir de données d'environ 340 horloges industrielles et prototypes répartis dans 72 institutions dans le monde. Le calcul effectué par le BIPM est le suivant : un algorithme fabrique l'Echelle atomique libre (EAL) comme une moyenne pondérée des horloges participantes. Cette échelle de temps est ensuite pilotée en fréquence par le BIPM grâce aux données d'étalons primaires de fréquence, qui réalisent la définition de la seconde du Système international d'unités SI. Une dizaine de laboratoires dans le monde possède de tels instruments, dont les données sont transmises régulièrement au BIPM. C'est notamment le cas du LNE-SYRTE, laboratoire pionnier dans le développement d'étalons de fréquence à atomes froids, qui dispose de trois fontaines au césium dont l'exactitude en fréquence représente l'état de l'art de ce type d'instrument. Le résultat du pilotage en fréquence de EAL est le TAI, auquel est ajouté ou soustrait un nombre entier de secondes pour réaliser l'UTC, afin d'assurer la concordance à $0,9 \mathrm{~s}$ près de l'UTC avec l'échelle de temps liée à la rotation de la Terre UT1 [1]. L'ajout ou le retrait d'une seconde intercalaire est décidé par le Service international de la rotation de la Terre (International Earth Rotation Service, IERS), dont le Bureau central est également intégré au SYRTE. Les résultats de calcul de l'UTC sont publiés au début de chaque mois par le BIPM dans sa Circulaire $\mathrm{T}$ et portent sur le mois écoulé. Pour les besoins pratiques de synchronisation, les LNM génèrent des prédictions en temps réel du futur UTC : pour la France, il s'agit de UTC(OP), signal physique issu d'une horloge industrielle située dans les locaux du LNE-SYRTE, dont la génération avait déjà fait l'objet d'une publication antérieure [2]. L'objectif de UTC(OP) est d'être le plus proche possible en temps du futur UTC. De plus, l'écart entre l'UTC et UTC $(\mathrm{OP})$ représente également le résultat de la comparaison clé qui permet le raccordement de la métrologie temps-fréquence française à l'organisation régionale européenne de métrologie Euramet. Cet article porte uniquement sur les résultats d'une modernisation récente de l'échelle de temps TA(F).

\section{Bref rappel historique du $\mathrm{TA}(\mathrm{F})$}

Dans un contexte national, le TA(F) est une échelle de temps «papier » jouant le rôle d'une référence de fréquence pour les laboratoires qui y contribuent. Il est calculé en temps différé à partir de données quotidiennes d'horloges commerciales au césium.

Initialement mis en œuvre au début des années 1970 à l'OP, le TA $(\mathrm{F}) \mathrm{a}$, pendant de nombreuses années, utilisé l'algorithme d'échelle de temps qui servait au calcul du TAI effectué à l'époque le Bureau international de l'heure $(\mathrm{BIH})$, et qui était également situé à l'OP : la différence entre les deux échelles provenait du nombre d'horloges qui y contribuaient, puisque contrairement au TAI, seules les horloges françaises servaient à générer le $\mathrm{TA}(\mathrm{F})$. À l'origine basé uniquement sur des horloges localisées à l'OP, le $\mathrm{TA}(\mathrm{F})$ a progressivement intégré des horloges 


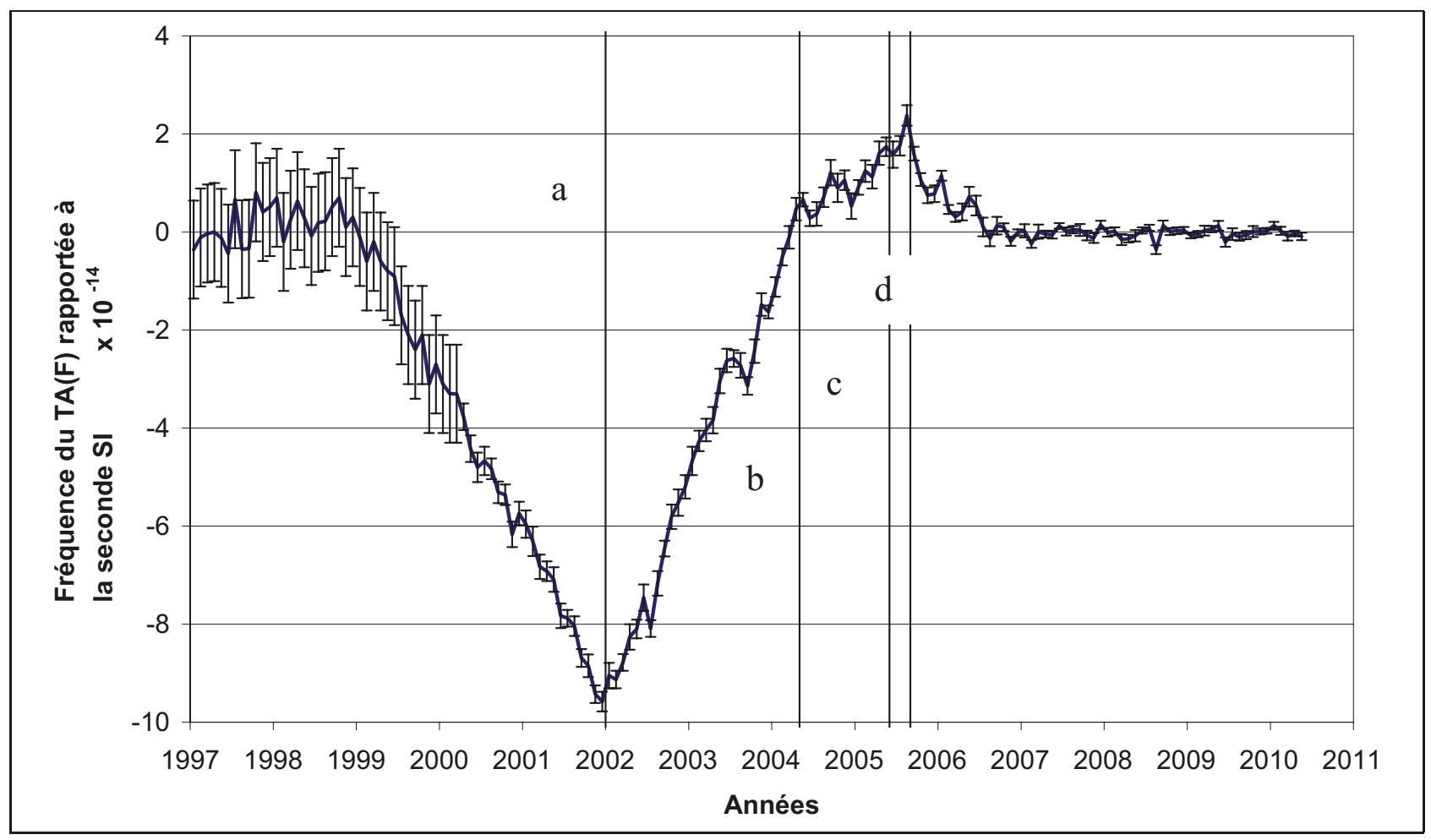

Fig. 1. - Fréquence du TA(F) rapportée à la seconde du SI. Les lignes verticales matérialisent les dates à partir desquelles : (a) un pilotage systématique mensuel a été appliqué ; (b) un pilotage systématique mensuel d'amplitude plus faible a été appliqué ; (c) un mélange entre un pilotage systématique et un pilotage basé sur des étalons primaires de fréquence a été mensuellement appliqué ; et (d) un pilotage mensuel uniquement basé sur les étalons primaires du laboratoire a été appliqué.

industrielles appartenant à différentes institutions ou entreprises françaises.

Au début des années 90, des études ont été menées dans le but de moderniser l'algorithme utilisé pour le calcul du TA(F). Mis en œuvre depuis 1997, l'algorithme utilisé pour le calcul du TA(F) est basé sur des modélisations Arima des horloges [3], Arima étant l'acronyme de «Autoregressive integrated moving average». Le principe de l'application au calcul du TA(F) peut être résumé de la manière suivante. Le modèle Arima de chaque horloge est d'abord construit à partir de l'observation pendant environ sept mois (215 d) des écarts de phase de l'horloge à l'ensemble. L'algorithme calcule l'échelle de temps à partir des écarts des données réelles d'horloges par rapport à leurs modèles. Ce calcul est effectué chaque mois sur la base de données quotidiennes des horloges participantes. Ces données d'horloges sont par ailleurs envoyées au BIPM qui les utilise comme contribution française au calcul du TAI. Les valeurs calculées du TA(F) sont également transmises au BIPM. Dans sa Circulaire T mensuelle, le BIPM publie les différences temporelles TAI - TA $(F)$ échantillonnées tous les cinq jours. Depuis 1997, la stabilité relative de ces différences TAI - TA(F), calculée sous la forme d'un écart type d'Allan sur une année de données, était de l'ordre de $3 \times 10^{-15}$ pour une période d'analyse de $30 \mathrm{~d}$. La variance d'Allan est une mesure de stabilité de fréquence relative normée bien adaptée aux différents types de bruits présents dans les oscillateurs et les échelles de temps.
Il est utile de rappeler ici que les deux échelles de temps TAI et TA(F) ont des finalités différentes : le TAI prend en compte toutes les horloges mondiales dont les données sont communiquées au BIPM, de manière à générer un intermédiaire de calcul qui conduit à l'UTC, le TA(F) a pour finalité de fournir aux organismes français participants une référence de temps fréquence aussi exacte et stable que possible sur des périodes d'analyses de $30 \mathrm{~d}$ et ce pour des applications à visées scientifiques.

La figure 1 montre l'écart relatif de fréquence entre le TA(F) et la seconde du SI. Cet écart relatif est calculé en deux étapes. D'abord, une interpolation linéaire est effectuée par la méthode des moindres carrés dans les données TAI - TA(F) publiées dans la Circulaire T, ce qui permet d'obtenir une fréquence moyenne entre ces deux échelles de temps sur la période couverte par une Circulaire T donnée. Ensuite, on additionne l'écart correspondant entre l'intervalle unitaire du TAI et la seconde du SI calculée par le BIPM en fonction des données d'étalons primaires de fréquence que les laboratoires ont communiquées dans le mois courant. Cet écart relatif est noté « $d$ » dans la Circulaire T, sans rapport avec le symbole d utilisé comme unité de jour. On peut voir sur la figure 1 que le TA(F), qui avait été recalé au $1^{\text {er }}$ janvier 1997 sur un des étalons primaires du laboratoire, exhibait une large dérive de fréquence jusqu'à fin 2001, où on atteint un écart relatif de fréquence proche de $-1,0 \times 10^{-13}$ entre l'intervalle unitaire du TA(F) et la seconde du SI. 


\section{Le Temps atomique français aujourd'hui}

En 2009, le TA(F) est calculé à partir de 26 horloges commerciales à césium, disséminées dans 9 institutions ou entreprises françaises. Ces différents organismes sont : Agilent Technologies (Palaiseau), le Centre d'essais des Landes (CEL, Biscarosse), le Centre d'essais de l'armement (Celar, Rennes), le Centre national d'études spatiales (CNES, Toulouse), la Direction des constructions navales (DCN, Brest), France Telecom R\&D (Lannion), l'Observatoire de Besançon (OB, Besançon), l'Observatoire de la Côte d'azur (OCA, Grasse) et l'Observatoire de Paris (OP).

Depuis une vingtaine d'années, les nombres d'horloges et d'institutions sont restés sensiblement les mêmes, et il ne semble pas raisonnable d'espérer un accroissement significatif du nombre d'horloges ou une amélioration notable de leur stabilité intrinsèque. De plus, au début des années 2000, la stabilité à long terme du TA(F) apparaissait limitée par sa dérive intrinsèque de fréquence, comme on peut le voir dans la première partie de la figure 1. D'un autre côté, le LNE-SYRTE dispose régulièrement des données provenant d'étalons primaires de fréquence qui réalisent la définition de la seconde du SI. Dans le double but d'assurer l'exactitude de l'intervalle unitaire du $\mathrm{TA}(\mathrm{F})$ et d'améliorer sa stabilité à long terme, il a été décidé en 2002 de piloter la fréquence de l'échelle de temps, dont la marche était laissée libre jusque-là, sur la fréquence des étalons primaires de fréquence du laboratoire. À cette époque, l'écart relatif en fréquence du $\mathrm{TA}(\mathrm{F})$ aux étalons primaires était du même ordre que son écart au TAI. Dans une première étape, décrite à la section 5 , la dérive du $\mathrm{TA}(\mathrm{F})$ a été lentement corrigée mensuellement par une fréquence choisie de manière à ramener le $\mathrm{TA}(\mathrm{F})$ vers le TAI. La valeur de cette correction a été calculée de manière à ne pas perturber la stabilité à $30 \mathrm{~d}$ du $\mathrm{TA}(\mathrm{F})$. Une fois la fréquence du $\mathrm{TA}(\mathrm{F})$ suffisamment proche de celle de la seconde du SI, au point qu'il devienne significatif d'utiliser les étalons primaires plutôt que le TAI pour calculer l'écart relatif de fréquence, le TA(F) n'a plus été piloté que par rapport aux quatre étalons primaires de fréquence du laboratoire. La section 6 décrit ce pilotage.

Les résultats sont dans un premier temps analysés en stabilité par rapport au TAI, plus stable sur les périodes considérées du fait d'un nombre d'horloges entre 10 et 15 fois supérieur. De plus, par le pilotage sur les étalons primaires du laboratoire, l'intervalle unitaire du TA(F) a été rendu proche de la seconde du SI. Dans toute la suite de cet article, sauf indication contraire, « seconde du SI » signifie «seconde du SI sur le géoïde en rotation, telle que calculée par le BIPM à partir des données d'étalons primaires qui lui sont communiquées », le géoïde en rotation étant défini par l'Union astronomique internationale (UAI) comme la surface équipotentielle gravitationnelle qui est la plus proche de la surface de la Terre. La validation de l'incertitude du raccordement du TA(F) aux étalons primaires du laboratoire peut ainsi être réalisée par comparaison avec cette seconde du SI.
Le pilotage du TA(F) décrit dans cet article est relativement indépendant des calculs du BIPM : si les étalons du LNE-SYRTE contribuent aux calculs du BIPM, ils ne sont pas les seuls, et par ailleurs l'algorithme utilisé par le LNE-SYRTE est, lui, totalement original. De plus, si les horloges françaises contribuent aux deux échelles de temps TA(F) et TAI, la corrélation entre les deux reste très faible, du fait du très grand nombre d'horloges qui contribuent au TAI. La validation des résultats du pilotage du TA(F) par le TAI et par la seconde du SI est donc justifiée.

\section{Pilotage en fréquence du TA(F) sur le TAI}

Fin 2001, l'écart relatif de fréquence du TA(F) par rapport aux étalons primaires du laboratoire était si grand qu'il pouvait être considéré comme similaire à son écart relatif au TAI à quelque $10^{-15}$ près. À cette époque, les procédures visant à piloter le $\mathrm{TA}(\mathrm{F})$ par rapport aux étalons primaires étaient en cours de développement, alors que l'écart entre le TAI et le TA(F) était disponible mensuellement dans la Circulaire T. Il fut donc décidé de choisir le TAI comme référence pour une première étape de ce pilotage, le but étant simplement ici de se rapprocher suffisamment de la seconde du SI pour que le pilotage sur les étalons du laboratoire devienne pertinent. À cet effet, la signification du nom de l'échelle de temps a également été précisée, en s'inspirant des notations du BIPM : depuis 2002, on note EAL(F) l'échelle atomique libre issue du processus ARIMA appliqué aux horloges françaises par l'algorithme de calcul de l'échelle, et $\mathrm{TA}(\mathrm{F})$ l'échelle de temps issue du pilotage en fréquence de EAL(F) sur les étalons primaires du LNE-SYRTE.

Dans la continuité avec les données existantes, une correction de fréquence a été appliquée mensuellement à EAL(F) pour piloter lentement le TA(F) vers le TAI : la correction de fréquence a été choisie la plus grande possible, mais suffisamment faible pour ne pas dégrader la stabilité à 30 d de l'échelle de temps. De janvier 2002 à avril 2004, cette correction mensuelle de fréquence était égale à $1,929 \times 10^{-16} \mathrm{~d}^{-1}$. De mai 2004 à mai 2005, le TA(F) s'étant rapproché du TAI, la correction mensuelle de fréquence a été réduite à $0,675 \times 10^{-16} \mathrm{~d}^{-1}$. Entre juin 2005 et novembre 2005, la correction de fréquence a résulté d'une combinaison entre une correction systématique et un pilotage sur les étalons primaires du laboratoire qui étaient disponibles à cette période. On peut voir sur la figure 1 le résultat de ces différents pilotages à partir de 2002. Depuis décembre 2005, le pilotage de EAL(F) est calculé uniquement à partir des données des étalons primaires du laboratoire.

\section{Pilotage en fréquence du $\mathrm{TA}(\mathrm{F})$ par les étalons primaires du LNE-SYRTE}

Dans une seconde étape, une fois le TA(F) rendu suffisamment proche de la seconde du SI, la correction de fréquence a été uniquement calculée à partir de l'écart relatif de fréquence entre $\operatorname{EAL}(\mathrm{F})$ et les étalons primaires 


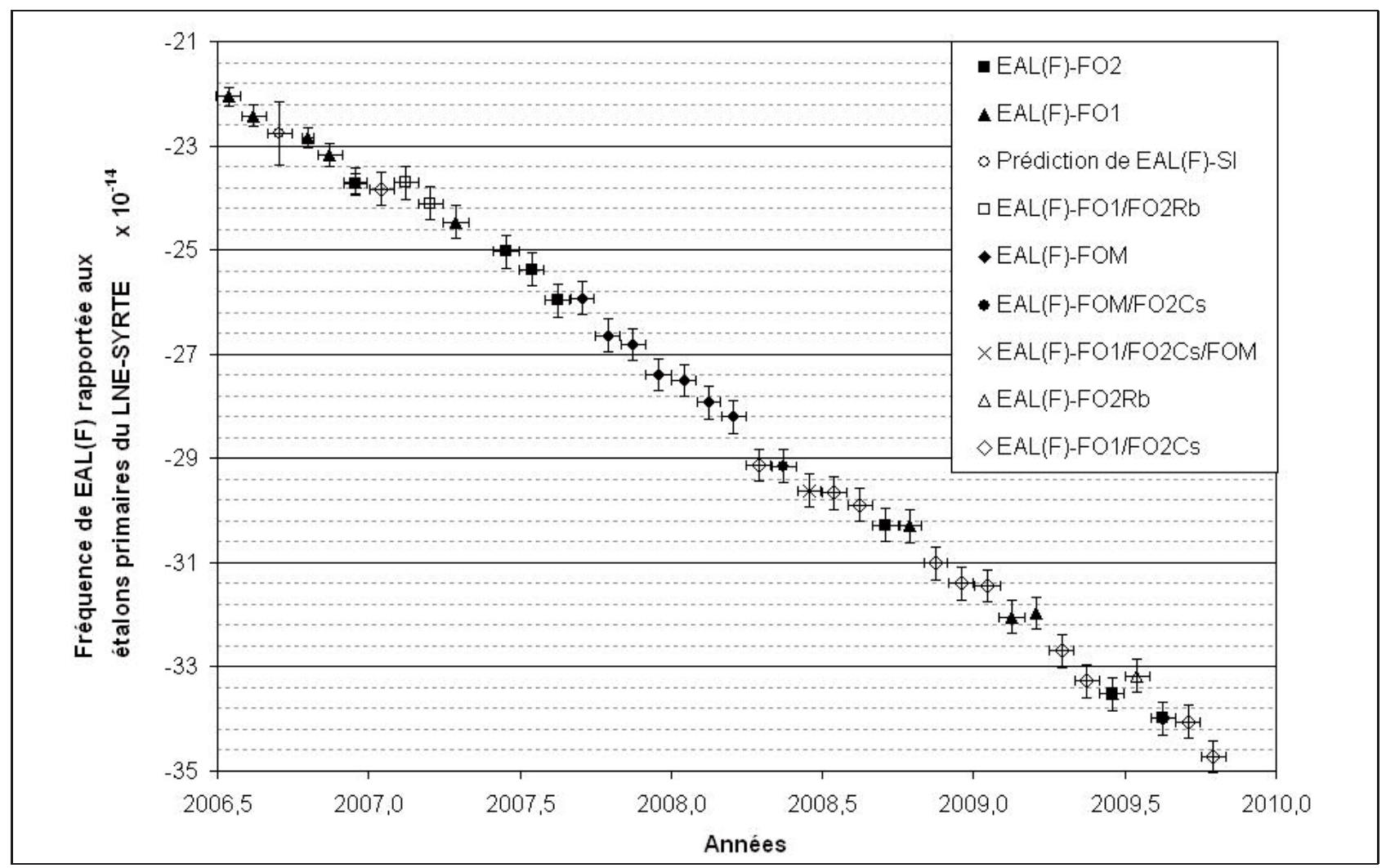

Fig. 2. - Fréquence de EAL(F) rapportée aux étalons primaires du LNE-SYRTE. Depuis 2006, on a utilisé des données provenant de FO2-Cs $(\bullet)$, FO1 $(\boldsymbol{\Delta})$, une moyenne pondérée de FO1 et FO2_Cs $(\diamond)$, une moyenne pondérée de FO1 et FO2_Rb ( $\square)$, FOM $(\diamond)$, une moyenne pondérée de FOM et FO2_Cs $(\bullet)$, ou une moyenne pondérée de FO1, FO2_Cs et FOM $(\times)$, sauf pour les points $(\circ)$ en septembre 2006 où une prédiction linéaire de l'écart relatif de fréquence entre le EAL(F) et la seconde du SI a été estimée, et en août 2008 où une prédiction linéaire de l'écart relatif de fréquence entre le EAL(F) et les étalons primaires FO1/FO2 a été utilisée. Les barres horizontales matérialisent, selon le cas, l'intervalle de temps pendant lequel les données des étalons primaires étaient disponibles pour calculer l'écart relatif de fréquence ou la durée de la Circulaire T utilisée pour la prédiction. Les barres verticales sont les incertitudes de ces évaluations pour $k=1$. Les valeurs utilisées pour le pilotage de EAL(F) correspondent simplement aux valeurs mensuelles lues sur l'axe des ordonnées avec un changement du signe.

du laboratoire. Ces étalons primaires sont actuellement au nombre de quatre :

- JPO (pour « Jet à pompage optique ») : un étalon classique à jet thermique de césium avec sélection et détection optique des atomes, dont l'exactitude actuelle est $6,3 \times 10^{-15}[4]$;

- FO1 (pour «Fontaine 1 ») : la première fontaine à atomes froids conçue pour des applications métrologiques, fonctionnant avec des atomes de césium, dont l'exactitude actuelle est $4,9 \times 10^{-16}$ [5];

- FO2 (pour «Fontaine 2 ») : la fontaine double, susceptible d'utiliser des atomes froids de césium (FO2_Cs) et/ou de rubidium (FO2_Rb), dont les exactitudes respectives sont actuellement $3,8 \times 10^{-16}$ et $4,7 \times 10^{-16}$ [5];

- FOM (pour «Fontaine mobile ») : la fontaine mobile à atomes froids de césium, dont l'exactitude actuelle est $6,6 \times 10^{-16}[5]$. Cet étalon avait été initialement conçu pour servir de prototype de faisabilité dans le cadre du programme spatial Pharao [6] développé par le CNES, un des instruments du programme spatial Ato- mic Clock Ensemble in Space (ACES) de l'Agence spatiale européenne. Cette horloge est actuellement le seul étalon primaire à atomes froids transportable dans le monde.

Il faut noter que FO2_Rb n'est pas à proprement parler un étalon «primaire», mais un étalon «secondaire» puisque la définition de la seconde est basée sur l'atome de césium. Mais dans le cadre de cette étude, nous utilisons le terme générique d'étalon primaire pour qualifier tous les étalons de fréquence dont les données sont utilisées pour le pilotage de notre échelle de temps. Par ailleurs, les données de ces quatre étalons sont transmises régulièrement au BIPM et constituent une contribution importante pour le calcul du TAI et la réalisation de la seconde du SI [7].

La figure 2 montre les évaluations de l'écart relatif de fréquence entre EAL(F) et les étalons primaires du laboratoire. Tous les étalons primaires ne sont évidemment pas toujours utilisables dans ce cadre. Mais depuis mi-2006, il n'y a eu que deux mois où il n'y avait pas au moins un étalon disponible. Lorsque plusieurs étalons primaires étaient disponibles, un écart relatif de 
fréquence moyen a été calculé à l'aide d'une pondération. Les poids sont inversement proportionnels aux incertitudes des liens entre les étalons primaires de fréquence et le maser à hydrogène qui leur sert d'oscillateur local. Ces incertitudes résultent de la somme quadratique de l'exactitude, de la stabilité et de l'incertitude de raccordement de chaque étalon [5,7]. Dans les cas où aucun étalon n'est disponible, une prédiction linéaire de l'écart relatif de fréquence entre le TA(F) et la seconde du SI est utilisée pour la correction de fréquence de $\operatorname{EAL}(\mathrm{F})$.

Les barres d'incertitude sur la figure 2 sont issues de la somme quadratique de deux termes. Le premier terme est l'incertitude, décrite ci-dessus, des liens entre les étalons primaires de fréquence et le maser à hydrogène : jusqu'à fin 2006, ces incertitudes sont calculées chaque mois. A partir de janvier 2007, la valeur typique de $0,9 \times 10^{-15}(k=1)$ a été retenue pour tous les étalons. Le second terme est une estimation de la stabilité relative de $\operatorname{EAL}(\mathrm{F})$ par rapport au TAI pour une période d'analyse de $30 \mathrm{~d}$ : la valeur typique qui a été choisie provient du calcul de l'écart type d'Allan $\sigma_{y}(\tau=30 \mathrm{~d})=3,0 \times 10^{-15}$, obtenue en 2001 à partir d'une année de données. La somme quadratique de ces deux termes conduit à une incertitude de $3,2 \times 10^{-15}(k=1)$. Cette valeur devrait pouvoir être diminuée grâce à l'amélioration de la stabilité liée au pilotage du TA(F) sur les étalons primaires. Lorsque l'écart de fréquence est calculé en l'absence de données d'étalons primaires, l'incertitude est calculée à partir d'une estimation conservatrice de la propagation de l'erreur de prédiction.

On peut également voir sur la figure 2 la dérive de la fréquence de $\operatorname{EAL}(\mathrm{F})$ par rapport à une moyenne pondérée des étalons primaires disponibles pour chaque évaluation. Sur un mois donné, le pilotage en fréquence de EAL(F) est simplement la valeur lue sur l'axe des ordonnées avec un signe inverse. Le résultat de ce pilotage de EAL $(F)$ a d'abord été évalué en terme de stabilité relative de l'échelle pilotée $\mathrm{TA}(\mathrm{F})$ par rapport au TAI. La stabilité de TAI étant d'environ un ordre de grandeur meilleure que celle de $\operatorname{EAL}(\mathrm{F})$, on a par conséquent simplement calculé l'écart-type d'Allan de TAI - TA(F), à partir des données publiées par le BIPM dans sa Circulaire $\mathrm{T}$ pour des points échantillonnés tous les cinq jours. La figure 3 montre les résultats obtenus en 2001, avant la mise en œuvre du pilotage, et en 2007 , la première année complète du pilotage en fréquence de $\mathrm{TA}(\mathrm{F})$ sur les étalons primaires du laboratoire. Pour une période d'analyse de $30 \mathrm{~d}$, soit environ $2,6 \times 10^{6} \mathrm{~s}$, on constate que l'on est passé d'une stabilité de $3,0 \times 10^{-15}$ en 2001 à $1,5 \times 10^{-15}$ en 2007, soit un facteur 2 d'amélioration. Pour des périodes d'analyses plus courtes, l'amélioration constatée est liée à une meilleure sélection des horloges contribuant à $\operatorname{EAL}(\mathrm{F})$, en attribuant un poids relatif plus élevé aux horloges les plus stables. La figure 3 montre aussi clairement que la dérive du $\mathrm{TA}(\mathrm{F})$ au-delà d'une période d'analyse de 30 d a été totalement éliminée, la stabilité à très long terme ne dépendant plus que de l'exactitude des étalons primaires et de leur raccordement à l'échelle de temps. L'objectif d'amélioration de la stabilité sur un mois et plus du TA(F) est donc bien atteint.

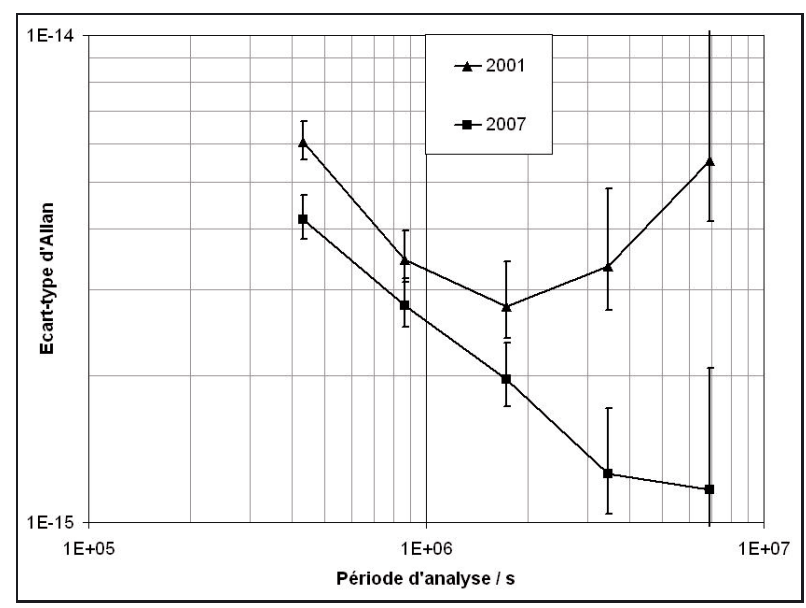

Fig. 3. - Stabilité de fréquence relative du TA(F) par rapport au TAI en 2001, avant la mise en œuvre d'un pilotage de l'échelle de temps, et en 2007, première année complète de pilotage de l'échelle de temps par les étalons primaires du LNE-SYRTE.

Lorsque l'on utilise la stabilité du TA(F) obtenue à partie de 2007 pour évaluer l'incertitude du raccordement aux étalons primaires, on obtient une incertitude de $1,8 \times 10^{-15}(k=1)$. C'est donc cette valeur qui caractérise l'intervalle unitaire du $\mathrm{TA}(\mathrm{F})$ depuis que le pilotage de l'échelle se fait uniquement à partir des données des étalons primaires du LNE-SYRTE.

\section{Validation de l'exactitude de l'intervalle unitaire du TA(F)}

Dans sa Circulaire T, le BIPM donne accès chaque mois à une évaluation de l'écart relatif de fréquence entre le TAI et la seconde du SI sur le géoïde en rotation. Cette seconde du SI est calculée sur la base des données d'étalons primaires communiquées par les différents LNM au BIPM. Elle représente ainsi un critère externe de validation de l'exactitude de l'intervalle unitaire du TA(F). L'écart relatif de fréquence entre le TAI et la seconde du SI est fourni par la valeur numérique attribuée au paramètre noté « $d$ » dans la section 4 de la Circulaire $\mathrm{T}$. Cette différence de fréquence moyenne est calculée sur les $30 \mathrm{~d}$ ou $35 \mathrm{~d}$ de référence d'une Circulaire $\mathrm{T}$ donnée, cette durée étant liée à l'échantillonnage sur $5 \mathrm{~d}$ des calculs du BIPM. La figure 4 donne le tracé de cet écart relatif moyen « $d$ » entre le TAI et la seconde du SI avec son incertitude combinée associée, tels que publiés dans la Circulaire T du BIPM depuis début 2007.

De manière analogue, la figure 4 contient également le tracé des écarts relatifs de fréquence entre le TA(F) et la seconde du SI. Ces points ont été obtenus à partir d'une interpolation linéaire par les moindres carrés dans les données TAI - TA $(F)$ fournies par le BIPM dans chaque Circulaire $\mathrm{T}$, ce qui aboutit à une fréquence moyenne pour chaque période donnée de 30 d ou 35 d. La comparaison du TA(F) à la seconde du SI est ensuite obtenue par addition du paramètre « $d »$ de la Circulaire $\mathrm{T}$ correspondante. Ici, les incertitudes associées sont obtenues par la somme quadratique entre l'incertitude combinée 


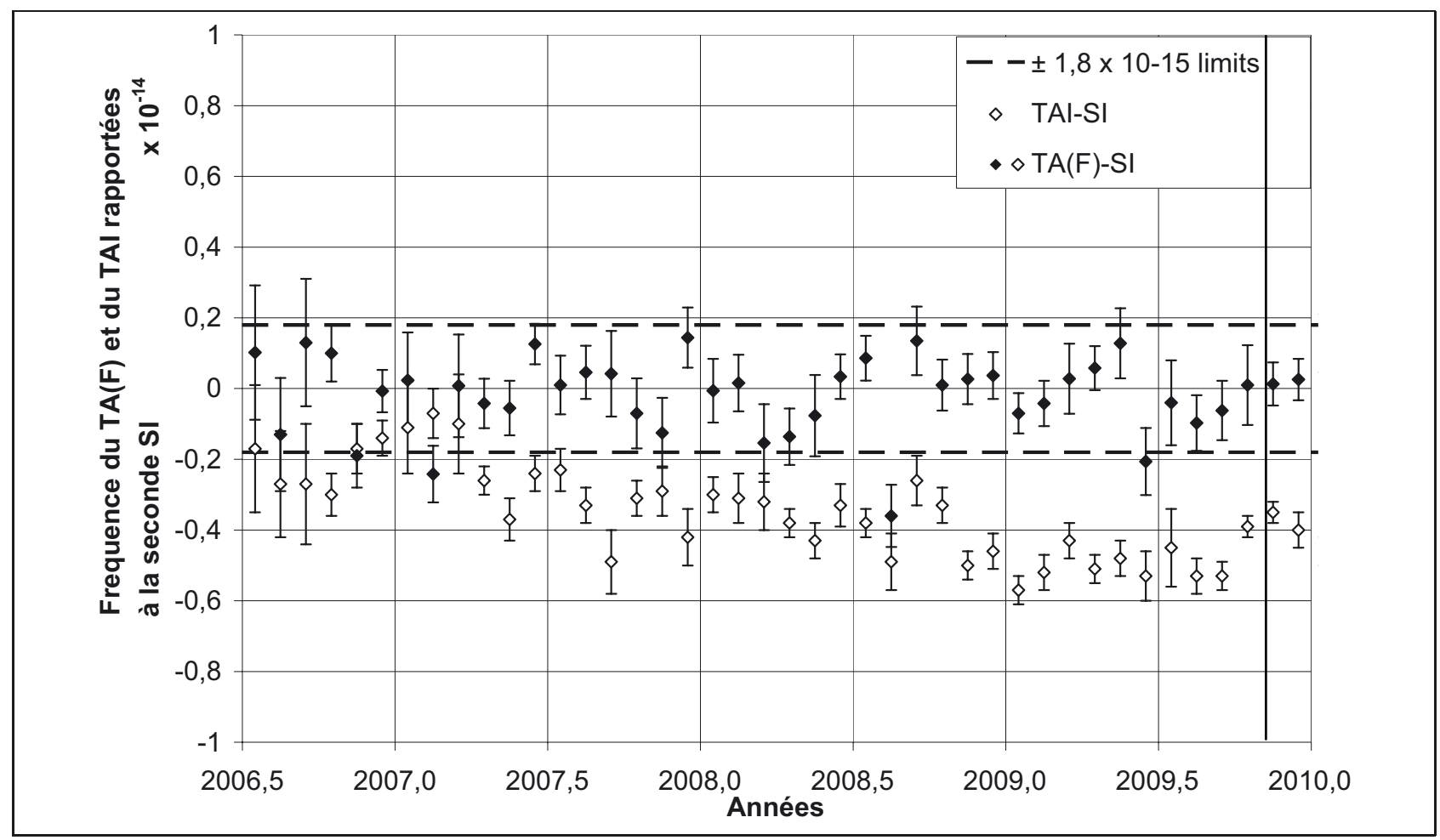

Fig. 4. - Fréquence du TAI et du TA(F) rapportée à la seconde du SI sur le géoïde en rotation telle que calculée par le BIPM : TAI SI représente simplement les valeurs du paramètre « $d »$, avec son incertitude combinée associée, publiées chaque mois par le BIPM dans sa Circulaire $\mathrm{T}$; $\mathrm{TA}(\mathrm{F})$ - SI est construit à partir du même paramètre « $d$ » duquel on retranche une fréquence moyenne obtenue par une interpolation linéaire par les moindres carrés dans les données TAI - TA(F) publiées dans chaque Circulaire T, l'incertitude étant dans ce cas la somme quadratique entre l'incertitude combinée associée à « $d$ » dans la Circulaire T et le résidu de l'interpolation linéaire.

associée au paramètre « $d »$ et le résidu de l'interpolation des données TAI - TA $(\mathrm{F})$ pour chaque Circulaire $\mathrm{T}$ : les barres d'incertitude sur TA(F) - SI sont donc toujours supérieures à celles de TAI - SI. Les incertitudes obtenues varient entre $0,5 \times 10^{-15}$ et $2,0 \times 10^{-15}$, dépendant aujourd'hui principalement des résidus de l'interpolation, liés à l'instabilité du TA(F).

Si un tel tracé ne peut être obtenu sans les données de la Circulaire T du BIPM, la réalisation actuelle du TA(F) est complètement indépendante des activités du BIPM. On constate que, depuis début 2007, l'écart relatif de fréquence entre le $\mathrm{TA}(\mathrm{F})$ et la seconde du SI est resté inférieur à $1,8 \times 10^{-15}$ en valeur absolue, à l'exception de trois points : deux de ces points en février 2007 et en juin 2009 sont très proches de cette limite statistique, un seul s'en écarte franchement. Ce point particulier correspond au mois d'août 2008, au cours duquel une coupure inattendue du réseau électrique a affecté en totalité le campus de l'OP. Le fonctionnement des horloges et des étalons primaires a été fortement perturbé, rendant tout étalonnage impossible pour le mois en cours. Ce point particulier a donc été obtenu à partir d'une prédiction de l'écart entre le TA(F) et la seconde du SI d'une part et entre le $\mathrm{TA}(\mathrm{F})$ et les étalons primaires FO1 et FO2 d'autre part. Sur la période considérée on peut donc dire que l'écart relatif de fréquence qu'on observe entre l'intervalle unitaire du TA(F) et la seconde du SI telle que calculée par le
BIPM valide le calcul d'incertitude à $1,8 \times 10^{-15}(k=1)$ sur le pilotage en fréquence du TA(F) de la section 6 . La qualité de cette réalisation repose sur les exactitudes des étalons primaires du LNE-SYRTE utilisés pour le pilotage de $\operatorname{EAL}(\mathrm{F})$, ainsi que sur l'incertitude du lien local permettant le raccordement de l'échelle de temps avec ces étalons primaires.

\section{Conclusion}

La transformation de l'échelle atomique libre TA(F) en une échelle de temps pilotée avait pour double objectif d'une part le rapprochement de l'intervalle unitaire de l'échelle par rapport à la seconde du SI, et d'autre part l'amélioration de la stabilité de l'échelle de temps sur une période d'analyse supérieure ou égale à un mois. Alors que l'ensemble d'horloges et d'institutions qui contribuent à l'échelle de temps est resté sensiblement le même au cours des années, le pilotage en fréquence sur les quatre étalons primaires du LNE-SYRTE a permis d'obtenir une stabilité relative du TA(F) par rapport au TAI de $1,5 \times 10^{-15}$ sur un mois, soit une amélioration d'un facteur 2 par rapport à la situation précédente où la marche de l'échelle de temps était laissée libre. De plus, la dérive a été éliminée sur le long terme, où la stabilité n'est plus fonction que de l'exactitude des étalons primaires et du raccordement de l'échelle de temps aux étalons. 
Opérationnelle depuis juillet 2006, la réalisation actuelle du $\mathrm{TA}(\mathrm{F})$ conduit à une échelle de temps dont l'écart avec la seconde du SI sur le géoïde en rotation, telle qu'elle est publiée par le BIPM dans sa Circulaire $\mathrm{T}$, est conforme à l'incertitude estimée du pilotage mis en œuvre : $1,8 \times 10^{-15}(k=1)$. Ainsi, l'intervalle unitaire du $\mathrm{TA}(\mathrm{F})$ réalise la seconde du SI dans ces limites. La qualité de cette réalisation dépend de l'exactitude des étalons primaires du LNE-SYRTE, dont les données sont communiquées régulièrement au BIPM, et surtout de l'incertitude du lien entre les étalons primaires et l'échelle de temps. Cette réussite majeure résulte de l'activité initiée au laboratoire dès 2002 sur les échelles de temps et de la qualité des étalons primaires du LNE-SYRTE. Il est prévu dans un futur proche de mettre en œuvre au LNE-SYRTE une nouvelle génération de UTC(OP), et une partie du pilotage en fréquence de ce signal s'appuiera directement des travaux présentés dans cet article.

\section{Remerciements}

Les auteurs remercient les institutions et entreprises situées sur le territoire national qui contribuent au $\mathrm{TA}(\mathrm{F})$ par la mise à disposition de leurs données d'horloges et de comparaisons.

\section{Références}

[1] GRanveaud M., «Échelles de temps atomique », Collection des monographies du Bureau national de métrologie, 11, éditions Chiron, 1987.

[2] Uhrich P. et al. «Le point sur la référence de temps française UTC $(\mathrm{OP})$ et raccordement du système européen de radionavigation par satellites EGNOS à UTC $(\mathrm{OP})$ », Bulletin du BNM, 1, 124, 2004, 25-35.

[3] ANDREUCCI C., "A new algorithm for the French atomic time scale", Metrologia, 37, 2000, 1-6.

[4] MaKdissi A. et Declerce E., "Evaluation of the accuracy of the optically pumped caesium beam primary frequency standard of the BNM-LPTF", Metrologia, 38, 2001, 409-425.

[5] BizE S. et al. «Réalisation et diffusion de la seconde au LNE-SYRTE fondées sur l'utilisation d'horloges en fontaines atomiques », Revue Française de Métrologie, 2, 18, 2009.

[6] LAURENT Ph. et al. "Design of the cold atom PHARAO space clock and initial test results", Appl. Phys. B, 84, 2006, 683-690.

[7] Les évaluations des étalons primaires du LNE-SYRTE qui ont été communiquées ces dernières années au BIPM, avec notamment 32 évaluations transmises par le LNESYRTE entre janvier et novembre 2009, peuvent être consultées sur le serveur FTP de la section Temps du BIPM, à l'adresse : http://www.bipm.org/jsp/en/TimeFtp. jsp?TypePub=data.

Article reçu le 18 décembre 2009, version révisée reçue le 9 décembre 2010. 